Marcos de Oliveira Lage Ferreira

\title{
Estruturas de Dados Topológicas Escalonáveis para Variedades de dimensão 2 e 3
}

Dissertação apresentada como requisito parcial para obtenção do grau de Mestre pelo Programa de Pós-graduação em Matemática Aplicada do Departamento de Matemática da PUC-Rio

Orientador: Prof. Hélio Côrtes Vieira Lopes 


\title{
Marcos de Oliveira Lage Ferreira
}

\section{Estruturas de Dados Topológicas Escalonáveis para Variedades de dimensão 2 e 3}

Dissertação apresentada como requisito parcial para obtenção do grau de Mestre pelo Programa de Pós-graduação em Matemática Aplicada do Departamento de Matemática do Centro Técnico Científico da PUC-Rio. Aprovada pela Comissão Examinadora abaixo assinada.

\author{
Prof. Hélio Côrtes Vieira Lopes \\ Orientador \\ Departamento de Matemática — PUC-Rio \\ Prof. Luiz Carlos Pacheco R. Velho \\ IMPA \\ Prof. Waldemar Celes \\ Departamento de Informática - PUC-Rio
}

Prof. Geovan Tavares dos Santos

Departamento de Matemática - PUC-Rio

Prof. Sinésio Pesco

Departamento de Matemática - PUC-Rio

Prof. José Eugênio Leal

Coordenador Setorial do Centro Técnico Científico - PUC-Rio 
Todos os direitos reservados. É proibida a reprodução total ou parcial do trabalho sem autorização da universidade, do autor e do orientador.

\section{Marcos de Oliveira Lage Ferreira}

Graduou-se em Licenciatura em Matemática na Universidade do Estado do Rio de Janeiro - UERJ

Ficha Catalográfica

Ferreira, Marcos de Oliveira Lage

Estruturas de Dados Topológicas Escalonáveis para Variedades de dimensão 2 e 3 / Marcos de Oliveira Lage Ferreira; orientador: Hélio Côrtes Vieira Lopes. - Rio de Janeiro : PUC-Rio, Departamento de Matemática, 2006.

v., 94 f: il. ; $29,7 \mathrm{~cm}$

1. Dissertação (mestrado) - Pontifícia Universidade Católica do Rio de Janeiro, Departamento de Matemática.

Inclui referências bibliográficas.

1. Matemática - Tese. 2. Estruturas de Dados Topológicas. 3. Topologia Computacional. 4. Geometria Computacional. I. Lopes, Hélio Côrtes Vieira. II. Pontifícia Universidade Católica do Rio de Janeiro. Departamento de Matemática. III. Título. 


\section{Agradecimentos}

Ao meu orientador professor Hélio Lopes pelo apoio e amizade durante a realização deste trabalho.

À CAPES e à FAPERJ pelos auxílios concedidos, sem os quais não poderia ter realizado este trabalho.

Aos meus pais Marcos e Lúcia, minha irmã Cynthia e minha namorada Viviane pelo carinho de sempre.

Aos meus colegas da PUC-Rio, em especial ao Thomas Lewiner.

Aos professores do departamento de Matemática, em especial ao Geovan Tavares, Paul Schweitzer, Marcos Craizer e Sinésio Pesco. Ao professor Marcos Alexandrino da USP e ao professor Luiz Velho do IMPA.

Aos funcionários do departamento de Matemática pela ajuda de sempre, em especial à Creuza. 


\section{Resumo}

Ferreira, Marcos de Oliveira Lage; Lopes, Hélio Côrtes Vieira. Estruturas de Dados Topológicas Escalonáveis para Variedades de dimensão 2 e 3. Rio de Janeiro, 2006.94p. Dissertação de Mestrado - Departamento de Matemática, Pontifícia Universidade Católica do Rio de Janeiro.

Pesquisas na área de estrutura de dados são fundamentais para aumentar a generalidade e eficiência computacional da representação de modelos geométricos. Neste trabalho, apresentamos duas estruturas de dados topológicas escalonáveis, uma para superfícies trianguladas, chamada CHE (Compact Half-Edge), e outra para malhas de tetraedros, chamada CHF (Compact Half-Face). Tais estruturas são compostas de diferentes níveis, que nos possibilitam alterar a quantidade de dados armazenados com objetivo de melhorar sua eficiência computacional. O uso de APIs baseadas no conceito de objeto, e de herança de classes, possibilitam uma interface única para cada função em todos os níveis das estruturas. A CHE e a CHF requerem pouca memória e são simples de implementar já que substituem o uso de ponteiros pelo de contêineres genéricos e regras aritméticas.

\section{Palavras-chave}

Estruturas de Dados Topológicas. Topologia Computacional. Geometria Computacional. 


\section{Abstract}

Ferreira, Marcos de Oliveira Lage; Lopes, Hélio Côrtes Vieira. Scalable topological data-structures for 2 and 3 manifolds. Rio de Janeiro, 2006. 94p. MsC Thesis - Department of Matemática, Pontifícia Universidade Católica do Rio de Janeiro.

Research in data structure area are essential to increase the generality and computational efficiency of geometric models' representation. In this work, we present two new scalable topological data structures, one for triangulated surfaces, called CHE ( Compact Half - Edge ), and the another for tetrahedral meshes, called CHF (Compact Half - Face ). Such structures are composed of different levels, that enable us to modify the amount of data stored with the objective to improve its computational efficiency. The use of APIs based in the object concept and class inheritance, makes possible an unique interface for each function at any level. CHE and CHF requires very few memory and are simple to implement since they substitute the use of pointers by generic containeres and arithmetical rules.

\section{Keywords}

Topological Data-Structures. Computational Topology. Computational Geometry. 


\section{Sumário}

\begin{tabular}{lll}
\hline 1 & Introdução & 11
\end{tabular}

\begin{tabular}{lll}
\hline 2 & Preliminares & 14
\end{tabular}

$\begin{array}{lll}2.1 & \text { Simplexos } & 14\end{array}$

\begin{tabular}{lll}
\hline 2.2 & Complexos Simpliciais & 15
\end{tabular}

\begin{tabular}{lll}
\hline 2.3 & Relações entre Simplexos & 16
\end{tabular}

\begin{tabular}{lll}
\hline 2.4 & Componentes Conexas & 18
\end{tabular}

$\begin{array}{lll}2.5 & \text { Variedades } & 18\end{array}$

2.6 Bordo de Variedades 20

$\begin{array}{lll}2.7 & \text { Relações Topológicas em Variedades } & 21\end{array}$

2.8 Programação Genérica 22

3 Trabalhos Anteriores $\quad 25$

3.1 Estruturas de Dados para Superfícies em $\mathbb{R}^{3} \quad 25$

3.2 Estruturas de Dados para 3-Variedades 31

3.3 Estruturas de Dados para $n$-Variedades 34

$3.4 \quad$ Estruturas de Dados para não-Variedades 36

4 A Estrutura de Dados CHE $\quad 38$

$\begin{array}{lll}4.1 & \text { Nível 0: Sopa de Triângulos } & 38\end{array}$

4.2 Nível 1: Adjacência entre Triângulos 40

4.3 Nível 2: Representação das Células 42

4.4 Nível 3: Representação das Curvas de Bordo 43

4.5 Exemplo de Construção da CHE 45

4.6 Interrogações Topológicas na CHE 46

$\begin{array}{lll}5 & \text { A Estrutura de Dados CHF } & 51\end{array}$

$\begin{array}{lll}5.1 & \text { Nível 0: Sopa de Tetraedros } & 51\end{array}$

5.2 Nível 1: Adjacência entre Tetraedros $\quad 55$

5.3 Nível 2: Representação das Células 56

5.4 Nível 3: Representação das Superfícies de Bordo 59

$\begin{array}{lll}5.5 & \text { Exemplo de Construção da CHF } & 60\end{array}$

$\begin{array}{lll}5.6 & \text { Interrogações Topológicas na CHF } & 61\end{array}$

$\begin{array}{lll}6 & \text { Comparações } & 66\end{array}$

$6.1 \quad$ Estruturas de Dados para Superfícies em $\mathbb{R}^{3} \quad 66$

E.2 Estruturas de Dados para 3-Variedades 69

$\begin{array}{lll}7 & \text { Conclusões e Trabalhos Futuros } & 73\end{array}$

\begin{tabular}{ll}
\hline Referências Bibliográficas & 92
\end{tabular} 


\section{Lista de figuras}

1.1 Gráfico performance $X$ memória. 11

1.2 Níveis da CHE e da CHF no gráfico performance $X$ memória. 12

2.1 Simplexos de dimensão $0,1,2$ e 3 em $\mathbb{R}^{m}$. 14

2.2 Exemplos de faces dos simplexos de dimensão 1,2 e $3 \mathrm{em} \mathbb{R}^{m} \quad 15$

2.3 Bordo e interior de simplexos de dimensão 1 e 2 em $\mathbb{R}^{m}$. 15

$\begin{array}{lll}2.4 & \text { Complexo simplicial } & 16\end{array}$

2.5 Complexo inválido 16

2.6 Estrela e Elo de vértices de uma esfera. 16

$\begin{array}{lll}2.7 & \text { 2-complexo regular } & 17\end{array}$

$\begin{array}{lll}2.8 & 3 \text {-complexo não regular } & 17\end{array}$

$\begin{array}{lll}2.9 & \text { Simplexos 1-adjacentes } & 17\end{array}$

$\begin{array}{ll}2.10 \text { Simplexos 0-adjacentes } & 17\end{array}$

$2.11 \psi$ e $\gamma$ são 0 -conectados e $\psi^{\prime}$ e $\gamma^{\prime}$ são 1-conectados. O complexo simplicial $\Sigma$ é uma 0-componente conexa. 18

$\begin{array}{ll}2.12 \text { Simplexos variedade } & 19\end{array}$

\begin{tabular}{ll}
\hline 2.13 Simplexos não-variedade & 19
\end{tabular}

$\begin{array}{ll}2.14 \text { Pseudo-2-variedade } & 19\end{array}$

$\begin{array}{ll}2.15 \text { 2-variedade } & 19\end{array}$

2.16 2-variedade combinatória orientada no sentido anti-horário. 20

2.17 Simplexos de bordo e interior de uma 3-variedade. 21

$\begin{array}{ll}2.18 R_{00}(\sigma) \text { e } R_{00}\left(\sigma^{\prime}\right) & 21 \\ 2.19 R_{10}\left(\sigma_{0}\right), R_{11}\left(\sigma_{1}\right) & \text { e } R_{12}\left(\sigma_{2}\right)\end{array}$

$2.19 R_{10}\left(\sigma_{0}\right), R_{11}\left(\sigma_{1}\right)$ e $R_{12}\left(\sigma_{2}\right) \quad 22$

2.20 Exemplo de uma $R B-$ Tree. 24

3.1 Nó surface. 26

3.2 Nó face 26

3.3 Nó half-edge 26

3.4 Half-edge de uma aresta de bordo. 27

\begin{tabular}{lll}
\hline 3.5 & Half-edges de uma aresta de interior. & 27
\end{tabular}

3.6 Nó Boundary Curve. 27

3.7 Nó edge 28

$\begin{array}{lll}3.8 & \text { Nó vertex } & 28\end{array}$

3.9 Vértice de interior. 28

3.10 Vértice de bordo. 28

3.11 Relação corner/half-edge. 29

3.12 Corners opostos $c$ e $o$. 29

3.13 Relação corner/directed-edge. 30

3.14 Informações armazenadas pela directed-Edge $\mathrm{e}^{d}$. 30

3.15 Nó 3-Manifold. 31

3.16 Nó Surface. 32

3.17 Nó Boundary Surface.

3.18 Nó Face. 32

3.19 Nó Half-Face. 32

3.20 Nó Edge. 33 
3.21 Nó Surface Edge. 33

3.22 Nó Half-Edge. 33

3.23 Nó Vertex. 34

3.24 Nó Surface Vertex. 34

3.25 Nós da Handle-Face.

3.26 Estrela de um $n m$-vértice não-variedade 37

3.27 Estrela de uma $n m$-aresta não-variedade 37

4.1 Nível 0 da CHE: Sopa de Triângulos. 38

4.2 Half-edges de uma aresta em seus triângulos incidentes. 39

4.3 Half-edges next he $_{\text {(he) e } \operatorname{prev}_{\text {he }} \text { (he). }} 40$

4.4 Relação entre vértices e half-edges. 40

4.5 Nível 1 da CHE: Adjacência entre os triângulos. 40

4.6 Half-edge oposta a half-edge $H E_{i d}$ he. 41

4.7 Nível 2 da CHE: Representação das células. 42

4.8 Escolha das half-edges armazenadas no contêiner VH[].

4.9 CHE nível 3: Representação das curvas de bordo. 44

4.10 Exemplo de construção da CHE. 45

5.1 Nível 0 da CHF: Sopa de Tetraedros.

5.2 Uma half-face de um tetraedro. 52

5.3 Relação entre half-faces e vertices. 53

5.4 Orientação das half-faces de um tetraedro $T_{i d}$ t. 54

5.5 Índice de uma half-edge. 54

5.6 Half-edge mate $\quad 55$

5.7 Half-edge radial 55

5.8 Nível 1: Adjacência entre Tetraedros.

5.9 Half-face oposta a half-face $H F_{i d}$. $\quad 56$

5.10 Nível 2: Representação das Células. 57

5.11 Half-face de bordo incidente a uma aresta de bordo. 58

5.12 Exemplo de construção da CHE. 60

7.1 Stanford Bunny, 100.000 vértices, 199.322 triângulos, CHE nível 2.79

7.2 Happy Buddha, 543.652 vértices, 1.087.716 triângulos, CHE nível2. 80

7.3 Modelo CSG, 82.020 vértices, 164.036 triângulos, CHE nível 1.

7.4 David, 50.329 vértices, 100.458 triângulos, CHE nível 3.

7.5 Dragon, 437.645 vértices, 871.414 triângulos, CHE nível 3.

7.6 $\quad$ Stanford Bunny, 48.810 vértices, 273.660 tetraedros, CHF nível 1.84

7.7 Stanford Bunny, classificação dos vértices CHF nível 2. 85

7.8 Stanford Bunny, classificação das arestas CHF nível 2. 86

7.9 Stanford Bunny, superfície de bordo CHF nível 3.

7.10 Hand, 28.793 vértices, 125.127 tetraedros, Campo Escalar. $\quad 88$

7.11 Blunto, 40.921 vértices, 187.318 tetraedros, CHF nível 3.

7.12 Gargoyle, 48.553 vértices, 258.229 tetraedros, CHF nível 3.90

7.13 Tempo de carregamento dos níveis CHE 91

7.14 Tempo de carregamento dos níveis CHF 91 


\section{Lista de tabelas}

4.1 Complexidade das funções resposta na CHE. 50

5.1 Orientação das half-faces de um tetraedro $T_{i d}$ t. 53

5.2 Complexidade das funções resposta na CHF. 65

6.1 Custo de memória para representação da topologia. 69

6.2 Custo de memória para representação da topologia. 72

7.1 Custo de memória para representação do modelo Stanford Bunny. 73

7.2 Custo de memória para representação do modelo Happy Buddha. 74

7.3 Custo de memória para representação do modelo CSG. 74

7.4 Custo de memória para representação do modelo David. 74

7.5 Custo de memória para representação do modelo Dragon. 75

7.6 Custo de memória para representação do modelo Stanford Bunny $\begin{array}{ll}\text { volumétrico. } & 75\end{array}$

7.7 Custo de memória para representação do modelo Hand. $\quad 76$

7.8 Custo de memória para representação do modelo Blunto. $\quad 76$

7.9 Custo de memória para representação do modelo Gargoyle. 